\title{
La transmisión de marca de las universidades españolas en sus portales webs
}

\author{
María Victoria CARRILlo DURÁN \\ Universidad de Extremadura \\ mvcardur@alcazaba.unex.es \\ Ana CAstillo DÍAz \\ Universidad de Extremadura \\ acasdia@alcazaba.unex.es \\ Tania Blanco SÁNCHEZ \\ Universidad de Extremadura \\ tania.blanco@e-campus.uab.cat
}

\begin{abstract}
Resumen:
Se parte de la idea de que la imagen y la comunicación correctamente gestionadas son activos estratégicos que potencian la marca y, en consecuencia, a la institución universitaria. La transmisión de marca de las universidades españolas a través de sus sedes webs se ha convertido para las universidades en un tema de gran importancia debido a la situación de incertidumbre en la que se encuentran las universidades. En este sentido, las sedes webs no solo son el lugar en el que los públicos tienen un primer contacto con la universidad, sino que además son una vía de comunicación con los públicos internos y externos, que permite garantizar a las instituciones una mayor competitividad, constituyendo un valor añadido para sus públicos.
\end{abstract}

Palabras clave: Marca; universidad española; sede web; marca online

\section{Brand transmission of spanish universities in their websites}

\begin{abstract}
:
It starts from the idea that the image and well-managed communication are strategic assets which enhance the brand and, consequently, to the university. Transmission brand of Spanish universities through their web sites has become for universities in an issue of great importance due to the uncertainty in which the universities are located. In this sense, the web sites are not only the place where the public have a first contact with the university, but are also a means of communication with internal and external audiences, which allows institutions to ensure greater competitiveness, constitute an added value to their audiences.
\end{abstract}

Key Words: Brand; spanish university; websites; branding online

\section{Referencia normalizada:}

Carrillo Durán, M. V.; Castillo Díaz, A.; Blanco Sánchez, T. (2013) La transmisión de marca en las universidades españolas en sus portales webs. Historia y Comunicación Social. Vol. 18 No Especial Octubre. Págs. 195-205

Sumario1. Introducción. 2. Conceptos de marca: actual, universitaria y online. 2.1. El concepto actual de Marca. 2.2. La Marca Universitaria. 3. Las sedes webs de las universidades españolas. 4. Metodología. 4.1. Metodología cualitativa. 4.2. Metodología cuantitativa. 5. Resultados 6. Conclusiones. 7. Bibliografía. 8. Anexos 


\section{Introducción}

Hoy en día las universidades españolas se están enfrentando a un contexto un tanto difícil como consecuencia de la crisis económica y los consiguientes recortes presupuestarios, la implementación del Espacio Europeo de Educación Superior (EEES), la creación de nuevas universidades, el aumento de la competencia nacional e internacional y la aplicación de las nuevas tecnologías de la información. A todo ello hay que sumar que las universidades españolas presentan un pobre reconocimiento de marca y competitividad a nivel internacional como lo demuestran los principales rankings internacionales de universidades (ARWU, The Times Higher Education World University Ranking, ect...).

Una de las principales causas por las que las universidades españolas tienen una marca débil se encuentra en la falta de interés por los denominados activos intangibles, como son la imagen, la cultura corporativa, la reputación,... Dichos intangibles pueden ayudar a diferenciar a las universidades y dotarlas de un valor distintivo frente a la competencia. Esta idea es apoyada por Fombrun (2001:273), que afirma que en una situación de crisis existen multitud de evidencias que demuestran que una buena imagen constituye un escudo gracias a su efecto amortiguador. Por tanto, estos elementos intangibles podrían ayudar a consolidar la imagen de la Universidad.

Por otra parte, todas las universidades españolas poseen un portal web en el que no solo disponen de información sobre sí mismas, sino que además utilizan este medio como herramienta para transmitir su marca y darse a conocer entre los usuarios.

No obstante, la transmisión de marca en el sector de las universidades apenas ha sido estudiada en el entorno online (Opoku, Hultman y Saheli-Sangari, 2008) y menos aún en España (Castillo, Carrillo y Tato, 2013). Por todo ello, en este trabajo se pretende analizar cómo las universidades españolas transmiten su marca online en sus sedes webs.

\section{Conceptos de marca: actual, universitaria y online}

\subsection{El concepto actual de marca}

Hoy en día la marca ya no sirve únicamente para distinguir un producto de otro, sino que representa algo más como valores, sueños, identidad, etc. A este respecto Temporal y Lee (2003) señalan que la marca es única y pervive en el tiempo; en cambio, el producto puede ser copiado por un competidor y queda rápidamente obsoleto. Estos autores resaltan la importancia de la marca como elemento emocional "los públicos compran un pensamiento, un sentimiento, una sensación física e incluso una interacción social, que produce una satisfacción placentera" (Temporal y Lee, 2003:39). 
Así, la marca es un fenómeno intangible que abarca las percepciones, actitudes y creencias. Se sitúa en un plano simbólico y emocional, hasta tal punto que algunos autores (Atkin, 2006 o Young \& Rubicam) consideran que la fe en las marcas ha reemplazado a la fe religiosa en la posibilidad de dar sentido a la vida humana e incluso estimulan el cerebro como religión ${ }^{1}$.

Se concluye así que la marca tiene un gran componente emocional y no solo funcional.

\subsection{La marca universitaria}

Respecto a la marca universitaria y siguiendo esta misma línea, Melewar y Akel (2005) explican que la marca universitaria está formada por tres valores emocionales: los atributos de marca, la autoestima y la implicación de las personas. Estos tres elementos emocionales son clave para crear la Marca Experiencia Universitaria. En el concepto de Marca Experiencia Universitaria priman las experiencias vividas en primera persona o las experiencias de otros públicos. Pero esta experiencia no solo está formada por el valor emocional, sino que es fruto de la unión de éste valor con otros. Según Villafañe (2004) los componentes de la Marca Universitaria Experiencia son:

El valor funcional. Lo constituyen los Recursos Entregables Demostrados (RED) que la universidad ofrece al estudiante y al resto de sus públicos.

El valor emocional. La Capacidad de Identificación y Empatía (CIE) de una persona con la universidad. En el ámbito emocional, la empatía es el principal valor emocional que la marca ofrece a su público.

El valor social. Implica un comportamiento positivo para con el entorno y diferenciado del comportamiento de otras universidades.

Estos tres valores satisfacen las expectativas de los públicos de manera eficiente. A través de ellos las universidades consiguen que sus objetivos y la promesa de marca ofrecida a sus públicos se puedan cumplir.

\section{Las sedes webs de las universidades españolas}

Las sedes webs universitarias puede ser consideradas como el vehículo más apropiado para gestionar la marca de las universidades por varias razones. La primera es el gran volumen de información que barajan las universidades y la necesidad de una gestión global de las herramientas comunicativas. La segunda es la escasa inversión económica necesaria y su alcance masivo. La tercera es que con frecuencia el primer contacto entre las universidades y sus públicos se realiza a través de la sede web.

1 Diario Público.20-05-2011. "Ser fan de Apple estimula el cerebro como la religión". 
Así, las sedes webs universitarias se han convertido en la tarjeta de presentación de las universidades, transmitiendo la comunicación de la institución y difundiendo la marca universitaria. Es el medio estratégico perfecto para difundir información y transmitir la Marca. Además con la aparición Web 2.0 las sedes webs universitarias han ampliando sus funciones, generando espacios de comunicación idóneos y actitudes colaborativas y creativas (Pérez, 2008), aumentando el número de de usuarios. De tal modo, que lo se comunica en las sedes webs y en las ramificaciones 2.0 afecta a la imagen y a la reputación universitaria. Por ello, en este artículo se estudia la transmisión de marca de las universidades españolas a través de sus sedes webs.

\section{Metodología}

Para conocer la transmisión de marca de las universidades españolas se ha utilizado una multimetodología. Se han empleado dos tipos de metodología científica: una metodología cualitativa y otra cuantitativa.

\subsection{Metodología cualitativa}

Para aplicar la metodología cualitativa se ha elaborado un panel Delphi formado por 16 expertos en comunicación universitaria (pertenecientes al ámbito académico e investigadores del área de comunicación, gestores universitarios y especialistas de la comunicación). El fin del panel era determinar las variables que transmiten las promesas de marca universitarias. Los expertos respondieron en dos ocasiones a un mismo cuestionario que estaba compuesto por 86 preguntas, de las cuales 85 correspondían a una escala likert y 1 consistía en jerarquizar de mayor a mayor. El motivo de que expertos contestaran en dos oleadas se debe a que en la segunda se llegó a un alto consenso (un rango intercuartílico por debajo del 10\%) y una cierta estabilidad (una variación del rango intercuartílico fijado en el 5\%).

Después se analizaron las respuestas del panel Delphi con el software PASW Statistics. Se calculó la media y el rango intercuartílico en cada uno de los ítems y se descartaron aquellos ítems que no cumplían con los requisitos de consenso (rango intercuatílico Q3-Q1 $=0<1$ ) y relevancia (puntuación media de 3 o más). Tras la eliminación de dichos ítems, se obtuvo el modelo válido de análisis de las promesas de la marca universidad online.

\subsection{Metodología cuantitativa}

Para aplicar el modelo de la Marca Universidad Online a las universidades españolas, se realizó un análisis de contenido de los mensajes emitidos por las universidades en sus sedes webs universitarias. Para llevar a cabo el análisis de contenido se establecieron los siguientes parámetros (tabla 1). 
Tabla 1. Parámetros empleados en el análisis de contenido

\begin{tabular}{|l|l|}
\hline Variables & $\begin{array}{l}\text { Son los aspectos genéricos que son evaluados y los cuales son desarrollados } \\
\text { en indicadores que dan la información necesaria de cada uno de estos grupos. } \\
\text { En este estudio las variables son: estudiantes, planes de estudio, docencia, } \\
\text { investigación, gestión económico-financiera, recursos y equipamiento, } \\
\text { innovación, internacionalización, prestigio, responsabilidad social } \\
\text { universitaria, empleabilidad, ciudad, relaciones con empresas y } \\
\text { autoevaluación. }\end{array}$ \\
\hline $\begin{array}{l}\text { Unidad de } \\
\text { análisis }\end{array}$ & $\begin{array}{l}\text { Constituye el objeto específico de estudio en una medición y se refiere al } \\
\text { objeto de interés en una investigación. En este artículo el objeto de estudio } \\
\text { son las páginas webs de las universidades españolas. } \\
\text { En estas páginas se analizan los mensajes textuales, visuales y audiovisuales, } \\
\text { mostrados. }\end{array}$ \\
\hline Indicadores & $\begin{array}{l}\text { Son los elementos concretos que evalúan y desarrollan cada una de las } \\
\text { variables establecidos para el análisis de la información. } \\
\text { En esta investigación los indicadores son: existencia de mensaje, ubicación, } \\
\text { adaptación de los mensajes a los grupos de interés, traducción, y refuerzo de } \\
\text { los contenidos de datos y audiovisuales. }\end{array}$ \\
\hline Item & $\begin{array}{l}\text { Es cada una de las unidades de las que se compone una prueba, un test, un } \\
\text { cuestionario. } \\
\text { En esta investigación existen dos grupos de ítems. } \\
\text { El primer grupo está formado por las posibilidades de respuesta del } \\
\text { cuestionario Delphi, como por ejemplo el ratio de profesores titulares en el } \\
\text { cuerpo docente. } \\
\text { El segundo grupo de ítems está compuesto por las posibilidades de respuesta } \\
\text { del modelo de análisis. En modelo de análisis hay 378 ítems, como por } \\
\text { ejemplo "Condiciones de acceso a los estudios universitarios indicador de } \\
\text { existencia de mensaje". }\end{array}$ \\
\hline
\end{tabular}

Fuente: Elaboración propia

De la combinación de los indicadores y las variables se definieron las promesas de marca de las universidades españolas transmitidas a través de sus sedes webs.

El procedimiento empleado para realizar el análisis de contenido de las sedes webs de las universidades españolas consta de tres partes. En primer lugar se observó cada una de las páginas webs de las 76 universidades españolas. A continuación se puntuó del 1 al 3 cada uno de los 378 ítems pertenecientes a las 14 variables y a los 6 indicadores de cada sede web universitaria.

En tercer lugar, partiendo de las puntuaciones obtenidas en el análisis de los mensajes en las sedes web, se realizó la ponderación de las variables, ya que se consideró que no todas las variables tienen la misma importancia (no es lo mismo obtener una buena puntuación en docencia que en ciudad, por ejemplo). Dicha ponderación se basó en las en la opinión vertida por los expertos el cuestionario del panel Delphi. Tras realizar la ponderación se obtuvo una puntuación en cada una de las universidades, que es el resultado del cumplimiento de la promesa de las marcas universitarias españolas a través de la información que publican en sus sedes webs. 


\section{Resultados}

De los resultados obtenidos tras la aplicación del modelo de promesa de marcar online en las universidades españolas llama la atención que ninguna universidad ha alcanzado el 6 de puntuación. Es decir, que las universidades españolas no transmiten de manera adecuada sus promesas de marca online. Solo 24 universidades (el $21,57 \%$ ) han conseguido un 5, la mitad de la puntuación ideal posible. 52 universidades (el 68,42\%) han logrado una puntuación inferior a 5, e incluso 4 universidades (el $5,26 \%$ ) no han llegado a una puntuación de 4.

A continuación la siguiente tabla (tabla 2) muestra la puntuación obtenida en cada una de las variables por la totalidad de las universidades españolas sobre un total de 10 puntos (que sería el ideal).

Tabla 2. Puntuación de las variables analizados en las sedes webs de las universidades españolas

\begin{tabular}{|l|c|}
\hline Variables & Puntuación sobre 10 \\
\hline Recursos & 5,448 \\
\hline Planes de estudio & 5,441 \\
\hline Estudiante & 5,307 \\
\hline Empleabilidad & 5,054 \\
\hline Investigación & 4,779 \\
\hline RSU & 4,756 \\
\hline Internacionalización & 4,735 \\
\hline Innovación & 4,627 \\
\hline Total & 4,443 \\
\hline Docencia & 4,350 \\
\hline Ciudad & 4,230 \\
\hline Prestigio & 4,150 \\
\hline Gestión & 3,821 \\
\hline Intercambio & 2,869 \\
\hline Autoevaluación & 2,647 \\
\hline
\end{tabular}

Fuente: Elaboración propia

Tras observar la tabla 2 se aprecia que los mensajes que mejor puntuación han conseguido son los relativos a las variables de recursos con una media de 5,448, seguido muy de cerca de los planes de estudio y los estudiantes. En cuarto lugar está la empleabilidad. El resto de mensajes presentan una media inferior a 5. Por orden descendente los que más puntuación han obtenido son investigación, RSU, 
internacionalización, innovación, docencia, ciudad, prestigio, gestión, relación con la empresa y autoevaluación. Los valores de estos dos últimos factores ni si quiera alcanzan el 3.

Respecto a la puntuación conseguida por los indicadores, en la tabla que se muestra posteriormente (tabla 3 ) se recopilan los puntos conseguidos por las universidades españolas.

Tabla 3. Puntuación obtenida los indicadores por las universidades españolas

\begin{tabular}{|l|c|}
\hline Indicador & Puntuación sobre 10 \\
\hline Existencia de mensajes & 7,671 \\
\hline Ubicación & 5,238 \\
\hline Adaptación a los stakeholders & 4,021 \\
\hline Traducción & 4,603 \\
\hline Refuerzo con datos & 4,338 \\
\hline Refuerzo multimedia & 3,439 \\
\hline
\end{tabular}

Fuente: Elaboración propia

En la tabla anterior se observa como el indicador que mayor puntuación ha conseguido es la existencia de mensaje con 7,671, una puntuación más que notable. En este sentido, es lógico que la existencia de mensaje sea la que mayor puntuación ha obtenido, ya que si un mensaje no existe no puede traducirse, adaptarse a otros idiomas, etc... El segundo indicador que más puntos ha logrado es la ubicación de los mensajes con 5,238 puntos. Los cuatro siguientes indicadores han obtenido menos de un 5 de puntuación. Estos son la traducción $(4,603)$, el refuerzo con datos $(4,338)$, la adaptación a los grupos de interés $(4,021)$ y el refuerzo multimedia $(3,439)$.

\section{Conclusiones}

Después de analizar los resultados obtenidos a nivel general en las universidades españolas, se concluye que los mensajes que transmiten las promesas de marca de las universidades españolas son poco contundentes y deberían expresarse con mayor nitidez.

En lo referente a la ubicación de los mensajes, se observa que con frecuencia la ubicación de los mensajes conlleva visitar diversas páginas para acceder a un contenido determinado.

Por otra parte, las universidades apenas refuerzan sus mensajes con datos y con elementos audiovisuales. Principalmente los refuerzos observados están relacionados con manuales corporativos, planes estratégicos, datos y cifras de la universidad. Se recomienda que las universidades empleen el refuerzo audiovisual ya que es más fácil de memorizar y crea un vínculo emocional superior con sus públicos. 
Respecto a la adaptación de los mensajes a los grupos de interés, las universidades españolas principalmente se centran en los estudiantes y en el PDI. Así, se puede afirmar que las universidades suelen subestimar a otros grupos de interés como son las empresas, los alumnos extranjeros, otras instituciones públicas, los egresados, etc... Sería más adecuado que las universidades dotaran a cada grupo de interés de información precisa y que esté de acuerdo con sus inquietudes. Esto se debe a que no todos los públicos están interesados en las mismas cuestiones.

En lo relativo a la traducción de los mensajes, se observa que las universidades españolas están preocupadas por la internacionalidad de su marca, gracias al EEES y a las becas de movilidad internacional. Aunque esto se contradice con la escasa traducción de los mensajes que realizan. Se ha constatado que más de la mitad de los mensajes referentes a las promesas de marca universitaria no son traducidos a otro idioma. Es más existen cuatro universidades $(5,26 \%)$ que no traducen de sus mensajes a ningún otro idioma. Además otras tres universidades $(4,48 \%)$ que apenas traducen sus mensajes al castellano. Así, la traducción de los mensajes es uno de los puntos pendientes en el que estas instituciones deben mejorar de manera significativa.

Los resultados acerca de la aplicación del modelo de promesa de marca universitaria evidencian que los valores en investigación e innovación son muy semejantes. Con asiduidad las universidades agrupan ambas variables en un mismo epígrafe debido a la asociación del concepto I+D+I (Investigación, Desarrollo e Innovación).

Se ha detectado una carencia de mensajes respecto a la variable de ciudad, sobre todo en las universidades online. Esto se debe a que no es necesario el desplazamiento de los estudiantes a la sede física. Así, la ciudad como promesa de marca en las universidades con enseñanza online no tiene razón de ser.

Las universidades españolas resaltan especialmente la variable de empleabilidad como promesa de marca universitaria. Esto es consecuencia de la alta tasa de desempleo juvenil (cercana al 60\%). Por ello, no es de extrañar que los estudiantes se inclinen por estudiar en universidades que entre sus características destaque la empleabilidad. Las universidades se han percatado de la importancia de la empleabilidad y casi todas tienen un servicio de orientación de empleo o inserción laboral. . No obstante, este hecho contrasta con la escasa puntuación que ha conseguido la variable de relación con la empresa. Esta paupérrima puntuación puede fundamentarse en que la mayoría de las universidades son públicas y hasta hace relativamente poco tiempo apenas existían acuerdos con empresas para desarrollar proyectos conjuntos.

En cuanto a la variable Responsabilidad Social Universitaria (RSU) prevalecen mensajes relativos a la igualdad de oportunidades entre hombres y mujeres y a la integración de los discapacitados. En este sentido, bastantes universidades han creado planes específicos para ayudar a estos colectivos.

La docencia, a pesar de ser la función fundamental de la universidad, no ha obtenido una buena puntuación en la clasificación. La pobre valoración se puede deber a que hoy en día los estudiantes aprecian más otro tipo de variables como por ejemplo 
la empleabilidad y las universidades tratan de dar respuesta a las preocupaciones actuales a través de los mensajes que exponen en sus sedes webs corporativas.

Una de las variables que menor puntuación ha obtenido en las universidades ha sido la gestión económico-financiera, sobre todo en la universidad privada. Esto último se debe a que la universidad privada, al no financiarse exclusivamente con fondos públicos, no tiene la necesidad de mostrar su gestión económica de manera pública. En cambio, las universidades públicas por el hecho de ser públicas deben mostrar una mayor transparencia y claridad en la gestión de sus presupuestos.

La variable de autoevaluación es la que peor puntuación ha conseguido respecto a su situación ideal. Esto puede ser fruto de la escasa tradición de las universidades españolas en autoevaluar su calidad. Además el propio sistema universitario español ya posee una agencia de control y evaluación (ANECA).

A nivel general, los resultados muestran que las universidades de titularidad pública han conseguido mejor puntuación que las universidades privadas en la transmisión de su promesa de marca online. Esto puede ser consecuencia de que la crisis económica golpea especialmente a las universidades públicas por la reducción de las ayudas y por el aumento de la competencia tanto a nivel nacional (con la creación de nuevas universidades) como internacional (con el EEES). Con dicho panorama las universidades públicas se han preocupado más por transmitir de manera correcta sus promesas de marcas online y mostrar las ventajas que brindan sus marcas.

Finalmente, los resultados del modelo de transmisión de marca universidad en las sedes webs revelan que las universidades españolas tienen marcas homogéneas y con posicionamientos parecidos, principalmente vinculadas a su titularidad. Se han identificado grupos de universidades con algunas características comunes, es decir que se detectado universidades con "identidades compartidas". Estos grupos de universidades con identidades semejantes son:

- Universidades privadas

- Religiosas

- Con carácter internacional

- Universidades españolas

- Mundo empresarial

- Vinculación local

- Nuevas tecnologías e $\mathrm{I}+\mathrm{D}+\mathrm{i}$

Así se concluye que apenas existen universidades que transmitan de manera novedosa su marca online, ya que la mayoría de las universidades se ciñen a los grupos que se han descrito anteriormente.

Este trabajo es fruto en parte de la financiación obtenida por el Gobierno de Extremadura y Fondos Feder.UE.

\section{GOBIERNO DE EXTREMADURA}

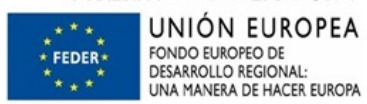




\section{Bibliografía}

ATKIN, D. (2006). El culto a las marcas. Barcelona: Robinbook.

CASTILLO, A.; CARRILLO, V.; TATO, J.L. (2013). "Branding online en las universidades españolas. Análisis de los valores funcionales y emocionales en sus websites corporativos". En Estudios sobre el mensaje periodístico, $\mathrm{n}^{\circ}$ Extra 19, 1, p. 85-97.

FOMBRUN, C. J. (2001). “Corporate reputations as economic assets”. En M. Hitt, R. Freeman and J. Harrison (eds.). Handbook of strategic management. Oxford: Blackwell.

MELEWAR, T.C.; AKEL, S. 2005, "The role of corporate identity in the higher education sector". En Corporate Communications: An International Journal, $10(1)$, p.41-57.

OPOKU, R.; HULTMAN, M.; SAHELI-SANGARI, E. (2008). "Positioning in Market Space: The Evaluation of Swedish Universities' Online Brand Personalities". En Journal of Marketing for Higher Education, 18:1, pp. 124-144.

PÉREZ, J. M. (2008): Teacher Training Curricula for Media and information Literacy. International Expert Group Meeting, en UNESCO Headquarters. Disponible en: http://portal.unesco.org/ci/en/files/27068/12133527103Background_Paper.doc/Background \%2BPaper.doc. [7-09-2013].

Público. 20-05-2011. "Ser fan de Apple estimula el cerebro como la religión". http:// www.publico.es/377424/ser-fan-de-apple-estimula-el-cerebro-como-la-religion. [10-09-2013].

TEMPORAL, P. y LEE, K.C. (2003). Branding de alta tecnología. México: McGraw Hill Interamericana Editores.

YOUNG \& RUBICAM. Brand Asset Valuator. Disponible en: http://young-rubicam. de/tools-wissen/tools/brandasset-valuator/?lang=en. [1-09-2013].

VILLAFAÑE, J. (2004). La buena reputación. Claves del valor intangible de las empresas. Madrid: Pirámide.

\section{Las autoras}

$\mathrm{M}^{\mathrm{o}}$ Victoria Carrillo Durán, Profesora Titular de Universidad de Comunicacion Audiovisual y Publicidad. Doctora en Ciencias de la información (Publicidad y RRPP) por la Universidad de Extremadura. Directora del grupo de investigación AR-CO (área de comunicación), de la Universidad de Extremadura y de la red internacional de grupos de investigación en Comunicación y Desarrollo (Real- Code). Ha dirigido numerosos proyectos de investigación del Plan Nacional, así como es miembro del Commitee Management de luna acción de investigación COST (2012), del Programa Marco, de la Unión Europea. Ha publicado diversos libros y capítulos relacionados con las influencias de la comunicación y la comunicación empresarial e institucional. Entre ellos se encuentran: "Comunicación integral y responsabilidad social en las organizaciones", Pearson (2009); Media and body cult, Pearson (2010), Communica- 
tion and Body Image, Pearson, Medios de comunicación y culto al cuerpo, Pearson (2013). Es autora de numerosas comunicaciones presentadas en congresos nacionales e internacionales como IAMCR, ECREA, ICORIA, AOM, AE-IC, WASET. Ha publicado artículos en revistas nacionales e internacionales indexadas en SCOPUS y JCR: Journal of Marketing for Higher Education, Global Journal Media, Observatorio Obs* Journal, El Profesional de la Información, Estudios del Mensaje Periodístico, Revista Latina de Comunicación Social, Saúde e Sociedade, Comunicación y Sociedad.

Ana Castillo Díaz, Doctora en Comunicación por la Universidad de Extremadura, donde trabaja como profesora contratada doctora en el área de Comunicación Audiovisual y Publicidad. Miembro del grupo de investigación AR-CO (área de comunicación), de la Universidad de Extremadura y de la red internacional de grupos de investigación en Comunicación y Desarrollo (Real- Code). Ha publicado diversos libros y capítulos relacionados con la comunicación empresarial e institucional. Entre ellos se encuentran: "Comunicación integral y responsabilidad social en las organizaciones", Pearson (2009); "Media and body cult", Pearson (2010) Es autora de numerosas comunicaciones presentadas en congresos nacionales e internacionales como IAMCR, ECREA, ICORIA, o los congresos de la Asociación Española de Investigación de la Comunicación (AE-IC). Ha publicado artículos en revistas nacionales e internacionales: Journal of Marketing for Higher Education, Global Journal Media, Observatorio Obs* Journal, El Profesional de la Información, Estudios del Mensaje Periodístico, Revista Latina de Comunicación Social, etc.

Tania Blanco Sánchez, Licenciada en Periodismo por la Universidad Autónoma de Barcelona. Diploma en Estudios Avanzados y doctorando en Comunicación Audiovisual y Publicidad en la Universidad de Extremadura. Ha realizado una internship en la United Nations Office to Support the International Decade for Action 'Water for Life' 2005-2015 UN-Water. Decade Programme on Advocacy and Communication. En dicha internship publicó entre otros: un Media toolkit sobre "Disaster prevention", un manual para periodistas "training programme of the Office" y un capítulo temático en "Water and cities" para el 2011 World Water Day. 